\title{
(Ent-)Hemmung bei Lacan
}

\author{
Andreas Spohn (Zürich)
}

Zusammenfassung: Lacan, oft verschrien als bis ins Absurde hinein intellektualisierend, kann nachgerade als Theoretiker des Affekts aufgefasst werden. Seine unorthodoxe Weiterführung von Freuds Dreifaltigkeit «Hemmung-Symptom-Angst» entwickelt zunächst ein um sechs weitere Haltungen erweitertes Diagnoseschema, das Patienten anhand typischer Angstvermeidungsmuster einschätzt. Hemmung bedeutet dabei motorischer, aber auch emotionaler Stillstand und hat am wenigsten Witz. Lacan korrigiert, dass die zu Freuds Zeitalter Gehemmten heute eher die "Verhinderten» seien, die «begehren, nicht zu können». Später behauptet er, dass Hemmung auftrete, wenn Bildhaftes zu sehr den Ablauf von Assoziationsketten störe. Heute ist Lacan vor allem mit der Bestimmung des Zeitgeistes als auferlegtem (und damit gehemmtem) Genuss aktuell. Immer wieder wird er von Kulturphilosophen als Stichwortgeber aufgerufen, wo sie nachvollziehen möchten, warum das Zeitalter des Gottestodes bzw. der Befreiung von Tabus paradoxerweise mit noch mehr Über-Ich-Strenge einhergeht. Lacan ist eher skeptisch, was Möglichkeiten einer wahrhaft befreienden Enthemmung angeht.

Schlüsselwörter: Lacan, Hemmung, Enthemmung, Hinderung, Wiederkehr des Verdrängten

Meint Hemmung negativ eine Art libidinöse Verstopfung, oder ist sie zum Glück immer da zur Stelle, «wo rohe Kräfte sinnlos walten», um sie zu kontrollieren oder gar in kulturell grossartige Leistungen zu veredeln?

Auf letzteres deutet Walter Mischels (2015) berühmtes Marshmallow-Experiment ${ }^{1}$ zur selbstdisziplinierten Impulshemmung hin, das auch als Voraussageinstrument für späteren Lebenserfolg gilt. Im Folgenden nutzen wir dieses Setting mit seiner Zuspitzung von Anmache und Verbot für Vignetten zu den typischsten hemmenden Abwehrformen.

Wie soll aber ausgerechnet Lacan, bekannt für die Konzeption des Unbewussten weniger als emotionalem Chaos, mehr als Sprachstruktur, zur Affekthemmung etwas zu sagen haben? Nun, das Unbewusste ist ihm keine sprachunab- 
hängige und darin schwer deutbare Entität, sondern Teil der individuell verfügten Sprache. Was sich als Affekt manifestiert, sei das Gleitend-Fragile der Sinnstiftung.

Wie Lacan dabei zu Hemmung und Enthemmung steht, soll hier nicht in philologischer Genauigkeit, sondern vor allem schlüssig, also mit dem Wagnis einiger Vorentscheidungen skizziert werden: Das abschreckende Begriffsarsenal der Lacan-Schule wird also abgerüstet, um das dort als irreführend gebrandmarkte schnelle, aber gegenüber wahren Zusammenhängen «gehemmte» Verstehen zu erleichtern.

\section{Freud: Ein «halbstarkes» Ich tut sich Zwang an - schützt so vor Angst und Symptombildung}

Schauen wir zunächst auf den Gründungsvater der Psychoanalyse, um entscheiden zu können, ob Lacan bei diesem Thema, wie ja generell von ihm reklamiert, zu Freuds Kerngedanken zurückkehrt, also dem Trieblichen volles Gewicht zumisst und es nicht kognitiv, «ich-psychologisch» verengt.

Für Freud haben die Auslöser der angstvollen Disposition im Unterschied zum bekannten «Fight-or-flight»-Paradigma Cannons nichts mit äusserer Bedrohung zu tun, sondern v. a. mit der Bedrohung des subjektiven Ganzheitsgefühls: dem Gefühl, dass Triebregungen nicht ungefiltert auslebbar sind.

Freud (1895d) selbst gab zu, dass seine erste Angsttheorie (Studien über Hysterie) «Verwirrung» stiften konnte. Damals hatte er die Angst als eine Art schreiendes Ablaufdatum beschrieben, das Alarm schlägt, wo nicht genossene Lustchancen bald den Enthaltsamen ungeniessbar machen. Das Subjekt flüchte dann nämlich in Verdrängung, in Symptome und Fehlleistungen, die man als eine Art Libidoverfaulung ansehen könnte. Hemmung taucht hier nur am Rande auf, als Assoziationsblockade bei hysterischer Erregung. (S. 188)

Mit der zweiten Theorie von 1926 (Hemmung, Symptom und Angst) wird die Angst dann vom Nebeneffekt der Verdrängung zum Signal einer antizipierten ödipalen Bedrohung, einerseits menschheitsgeschichtlich vererbt, andererseits individuell interpretiert. Denn jetzt ist es die Instanz des Ich, welche sozialverträgliche Reaktionen auf solcherlei «Arbeitsanforderungen» prüfen muss (woher es genau weiss, was genehm ist und was nicht, bleibt jedoch wie zuvor unklar).

Die beste Abwehr Gefahr verheissender Impulse ist nun jedenfalls nicht mehr die Verdrängung, sondern die vom Ich noch bewältigbare Hemmung - das Ich findet damit freilich selbstversteckte Ostereier bzw. entschärft selbstgelegte Bomben: Es erkennt erst die Erotisierbarkeit einzelner Funktionen und ist alsdann stark genug, sie «normalneurotisch» aufzuhalten (eine schwache Leistung jedoch, 
dass es sich damit selbst hemmt). ${ }^{2}$ Immerhin vermeidet Hemmung sowohl die Verdrängung als auch das alternativ zu ihr sich bildende Symptom, eine kreative Kompromissbildung, die sich metaphorisch eine Anpassung erfindet, die aber ersatzweise so befriedigend sein kann, als sei der Trieb ausgelebt worden. ${ }^{3}$

Während das Ich erst erotisiert und dann hemmt, also etwa in Marshmallows reizende Körperteile sähe und daraufhin den Appetit verlöre, würde die Symptombildung der Essfunktion «pathologisch» einen unschuldigen Sinn abringen und beispielsweise notorisch Kaugummi kauen.

Die Hemmung kann für Freud verschiedene Ursachen haben, den 1923 neu erdachten topologischen «Instanzen» Es und Über-Ich zugemessen:

1. Hemmung, um Es-Konflikten auszuweichen, nachdem Situationen oder Personen und insbesondere Körperteile erotisiert ihre unschuldige Alltäglichkeit verloren haben (typischerweise eine motorische Hemmung, z. B. weil Laufen neu der Vergewaltigung von «Mutter Erde» gleichkäme)

2. Hemmung, um Über-Ich-Konflikten auszuweichen, nachdem paradoxerweise sogar hehre Ziele ihre Unschuld verloren haben (typischerweise eine zwangsneurotische Denkhemmung, z. B. weil das forschende Eindringen in die Materie neu mit Penetration assoziiert wäre). ${ }^{4}$

3. Hemmung in Folge von Überanstrengung, nachdem das Ich lange solchen Konflikten mit dem Es oder Über-Ich ausgewichen ist. Es kann also auch insgesamt «ausbrennen» (typischerweise eine Depression). Dies wäre eine Funktionshemmung nicht aus Vorsicht, sondern durch anderweitiges Überinvestment: «Wie ein Spekulant, der seine Gelder in seinen Unternehmungen immobilisiert hat». (Freud, 1926d, S. 11)

All diese Hemmungen auflockern könne der Humor, scheint doch die Lust am Witz «aus erspartem Hemmungsaufwand hervorzugehen». (Freud, 1905c, S. 204)

Fazit: Hemmung ist für Freud indirekter Ausdruck von Erregung, Angst gilt ihm dagegen zunächst als Zeichen für die Verdrängung von solcherlei erregenden Gefühlen bzw. von Sexuellem. Später wird Angst spezifischer als ödipale Gefahr erahnt. Das darauf spezialisierte Zensur-Ich kämpft gegen Sexualisierbares, vermeidet es bzw. flüchtet. Subklinisch gilt die Hemmung v. a. der Vermeidung von bestimmten Bewegungen: Sie stoppt Triebdurchbrüche im Alltag, stoppt Angst machende Triebverdrängungen (wobei die Abkehr-Bewegung zugleich die Wiederkehr-Bewegung des zu Verdrängenden sein kann) und stoppt die metapho- 
rische Bewegung der Triebumbildung im Symptom. Die Hemmung wäre damit sozusagen der unkreative, schlichtweg verzichtende Bruder des oft verhaltensauffälligen Symptoms.

\section{Lacan (1962-1963): Hemmung ist symptomhinderndes Ohnmachtsbegehren}

Lacan, der das Unbewusste erst als Gesellschaft und Sprache in uns erklärt, es dann gar mathematisiert, liefert einen derart verhackstückelnden formellen Remix Freuds ab, dass kaum zu sagen ist, wo genau er zwischen ganz frühem und spätem Freud steht. Ohnehin gefällt sich Lacan oft im Umsturz psychoanalytischer Glaubenssätze: Angst etwa habe man weniger vor dem Verlust jenes gewissen Etwas, das andere temporär zu verführen und zu binden vermag, sondern davor, sich andere als dauerverbunden erträumen zu müssen (vgl. Anmerkung 5).

Im Seminar 10 von 1962/1963 kommt Lacan (2009) auf Freuds Gruppierung Hemmung-Symptom-Angst zu sprechen. Hier entfaltet er auch nochmals die zum Verstehen seiner Lehre so wichtige Idee des in Verlusterfahrungen ${ }^{5}$ gebildeten, später aber apriorischen Objektbeziehungs-Phantasmas: Das Objekt der Begierde sei nämlich mitnichten uns affizierend da draussen, vielmehr stecke es in uns, in unserer stets unbewusst erotisierenden, auch scham- und angstvollen Wahrnehmung. Realitätsobjekte gebe es nur als Glaubensobjekte einer Art Privatideologie. Stets mische sich in die Objektwahrnehmung eine die Verlusttraumata verarbeitende Sinnstiftung. In anderen Worten: Das Phantasma des Verlorenen prägt die Art und Weise, wie gehemmt ein Subjekt sich an den Anderen - ein inneres Erwartungsschema - wendet. Ganz grundsätzlich geniesst es dabei männlich codiert (indem es v. a. mit Gadgets davon ablenkt, dass es etwas zu verlieren hat) oder aber weiblich codiert (per starkem Glauben an ein Unverlierbares).

Lacan preist diese Prägung der Wahrnehmung durch eine unbewusste «Verlustaversion» als seine grosse Erfindung: Für ihn gibt es vor oder hinter dem sinnstiftenden Phantomobjekt keine kognitiv isolierbaren, dem Subjekt entgegenstehenden Objekte, von denen ich-psychologisch wie im Werbeslogan «Sind sie zu stark, bist du zu schwach» ein Anpassungsdruck ausgehen müsste. Er bezeichnet dieses höchst subjektive Phantom, dieses in entwicklungspsychologischen Verlusten kreierte Skript zur Sinnaufladung der Objekte als eine privatideologische «Objektursache» (die bei ihm sogar ganz mit seinem Objekt, dem berühmt-berüchtigten «objet a» zusammenfällt).

Wenn für Freud, so mag sich Lacan gedacht haben, in der Not des Triebdrucks die Hemmung die «leichtere», unpathologischere Reaktion ist (v. a. verglichen mit 
Panikattacken oder mit der Symptombildung), dann liesse sich doch ein ganzes Tableau nach Graden des Bewegtseins und der Schwierigkeit aufstellen. Er fährt nun eine komplexe Neunermatrix auf, alles ausgerichtet auf Triebabwehr, mehr oder weniger bewegt (enthemmt) und schwierig (undeutbar) sich dem Zentralaffekt Angst bzw. dem von den subjektiven Verlusten geprägten Objekt anzunähern. Lacan erweist sich hier als Theoretiker einer angemessenen Distanz zum bedrohlich kreativen Teil der eigenen Entwicklungstraumata. Jeder Mensch, jedes Subjekt habe bevorzugte Positionen hierzu (relativ unabhängig von Einordnungen durch die ICD oder nach psychischen Strukturen). ${ }^{6}$ Diagnostisch wertvoll sei es aber auch, den «Fingerabdruck» des Subjekts in typischen Abfolgemustern zu beobachten, etwa von Hinderung zu Aufruhr oder von Empörung und Gefühlsbewegung zu plötzlichem Impulsdurchbruch (passage à l'acte).

Abbildung 1 Tableau «Angstnahe Distanzierungen zur Angst, im Symptom und in der Hemmung»

Schwierigkeit $\rightarrow$

\begin{tabular}{|c|c|c|c|}
\hline $\boldsymbol{H}$ & $\begin{array}{l}\text { Hemmung } \\
\text { Begehren, nicht zu sehen }\end{array}$ & $\begin{array}{l}\text { Hinderung/Ohnmacht } \\
\text { Begehren, nicht zu können }\end{array}$ & $\begin{array}{l}\text { Verlegenheit/Empörung/Bedrängnis } \\
\text { Der Begriff Angst }\end{array}$ \\
\hline Bewegung $\Downarrow$ & $\begin{array}{l}\text { Gefühlsbewegung/Verkennen } \\
\text { Begehren, nicht zu wissen }\end{array}$ & Symptom/Allmacht $\mathbb{V}$ & $\begin{array}{l}\text { appelfreie, plötzlich-suizidale } \\
\text { passage à l'acte }\end{array}$ \\
\hline$A$ & Aufruhr/Ichideal & $\begin{array}{l}\text { (appelatives, trauerndes) } \\
\text { Ausagieren/acting-out }\end{array}$ & $\sqrt{ }$ Angst \\
\hline
\end{tabular}

Vor allem die obere Zeile in Abbildung 1 soll hier interessieren (Lacan 2009, Sitzung 14.11.1962), steht sie doch unter dem Titel «Hemmung» - wie bei Freud verstanden als Lähmung der normalen motorischen Bewegung, explizit aber auch der emotionalen Bewegung.

Bei der eigentlichen Hemmung meint der Untertitel «Begehren, nicht zu sehen» das Übersehen von individuell Gewünschtem, sozial aber Unerwünschtem: ${ }^{7}$ Das Irritierende am erwähnten, Traumata reenactenden Skript zur Sinnstifung ist hier ausgeblendet. Schon im Ansatz wird also ein Einstehen für die Objektursache, anders gesagt für die «erotischen Menschenrechte» (so Blanchot 1947 zu Sades Imperativ, sich ungehemmt des Körpers zwecks Luststeigerung zu bedienen) ausgebremst, werden Verdrängung und Symptombildung vermieden - wie auch jede weitere hier von Lacan erwogene Weise, «Erregtheiten» überhaupt zu registrieren, zu verarbeiten oder zu zeigen.

Hören, um nicht zu hören, sehen, um nicht zu sehen: Es hat viel von dem, was Melvilles Kurzgeschichte Bartleby mit dem notorischen «Ich möchte lieber nicht» in extremis veranschaulicht. 
So kann die Hemmung durch ihre radikale Triebunterdrückung gestörter anmuten als das Symptom, das immerhin den Impuls noch anerkennt, um sich erst dann ins Metaphorisieren zu flüchten. Andererseits rafft sie sich oft noch auf zum Aufsetzen einer triebbereinigten Alltagsmaske - zu verstehen als Einbehalten der eigenen Münze zwecks ambivalenter Wertsteigerung, mit viel Fassade, falschem Selbst und etwas rigiden Beziehungsformen.

Lacans gehemmter Marshmallow-Proband würde wohl entweder gar nicht erst antreten oder er sässe appetitlos vor dem Mäusespeck.

Lacan behauptet nun, Theoretiker sprächen noch immer von «Hemmung», wo man im Alltag doch längst eher von der «Hinderung» geplagt sei, welche die Sexualisierung oder abstrakter die Konfliktzuspitzung immerhin noch zur Kenntnis nimmt. Und es ist ja tatsächlich «schwieriger», sich in Richtung angstvolle Erregung zu begeben. Wobei es auch symptomatisch für diese Selbsthinderung wäre, die Bewegung alsbald mit der manierierten Kreativität eines Museumskurators tarnen zu müssen (Harari 2001, S. 20 f). Das Subjekt hangelt sich alsdann sozusagen von stehendem Ausdruck zu stehendem Ausdruck, was sich auch in Lacans späterer Idee eines «Bürokratiediskurses» wiederfindet.

An die Stelle des Stillstands bei Ödipus-Signalen setzt Lacan unterschiedlich starke Abbremsungen bei drohender Selbstbegegnung. Bei der Hinderung etwa wird diese kaschiert durch die geradezu lustvoll aufgesuchte Sicherheit des «Man»: Ein Begehren, nicht zu dürfen, was man will, und zu müssen, was man zivilisiert eben so tut (also gewissermassen dem im Museum bereitgestellten Audioguide nachzuplappern, statt den Mut zu haben, sich seines eigenen «Begehrens» zu bedienen).

Wenig ist da erlaubt: «Nicht können» mag zwar peinlicher sein als «gar nicht wollen», aber es muss ja keine eigene Unfähigkeit zugegeben werden, wo andere oder eine allzu harte Realität hinderten. Als Teil des Skripts der Objektursache wird die Hinderung zum geradezu eigenständigen, zwanghaft verfolgten Begehren, nicht zu können. ${ }^{8}$ Unter dieser Rubik wären narzisstische Störungen zu vermuten.

Die Marshmallows werden natürlich auch gehindert nicht verzehrt, aber das überschüssige Begehren, nicht zu können, führt z.B. zu Vorab-Bekundungen, wie schlecht man sich zusammenreissen könne oder wie unklar der Versuchsleiter in seinen Instruktionen geblieben sei. So ist der Kontrast hergestellt, um danach die «Prüfung» umso glatter, museumsreifer bestehen zu können.

In verlegener Empörung (höchster Schwierigkeitsgrad in der Hemmungszeile) traut sich das Subjekt mehr, kann sich nun mit der Objektursache des eigenen Begehrens konfrontieren, bremst sich erst im letzten Moment vor einem 
«Szenemachen» aus. Jedoch ist das Subjekt verlegen, zu sehr bleibt das sinnstiftende und auch Worte auf die Zunge legende Objekt buchstäblich verlegt (Widholm 2008, S. 35). Ein gehemmtes, quasi kaltes Echauffieren in Folge eines bedrohlichen Annäherns an die Dramatik des inneren Skripts, Indiz einer im Sinne Kierkegaards überdimensioniert erkannten, allzu sehr «begriffenen» Verantwortung für die Freiheit. Diese erzeugt eine stumme Empörung gegen wieder nicht wegzudenkende, insgeheim gar verantwortlich gemachte andere (eigentlich gegen das eigene Schema von ihnen, abstrakter gegen die Unverfügbarkeit der zu ihnen reichenden Sprache). Passiv-aggressives Verhalten liegt hier nicht allzu fern.

Der Proband tritt wiederum nicht wirklich ins Spiel ein, setzt sich aber mit Anzeichen des Heisshungers erst angespannt, dann entmutigt seufzend vor die Verlockung. Eventuell wirft er am Ende trotz des «Erfolges» den Versuchsleitern Scheinheiligkeit vor.

Von den weiteren Positionen der Abbildung 1 seien noch die beiden «enthemmten», paniknahen Aktpositionen kurz kommentiert.

Das Acting-out ${ }^{9}$ ergreift das sonst eher Zuschauer-artig passive Subjekt, das genug der (sein Trauma ignorierenden) Worte hat und zur reenactenden Objektursachen-Aufführung auf die Bühne springt.

Die Passage à l'acte (eigentlich die französische Übersetzung des Actingout) wird von Lacan differenzierend für die gegenläufige Situation reserviert: ein Agieren, das plötzlich ganz den Mut zur Aufführung verliert. Der eigentliche Akt besteht darin, dass das Schauspieler-Subjekt den Rahmen verlässt, sich wie um vor Scham im Boden zu versinken von der Bühne wirft.

Nicht enthalten im Tableau sind folgende nur leicht enthemmte Haltungen:

, Der Witz etwa treibt nicht hemmend ab, sondern gebärt ironisierend. Paradigmatisch ist er ein kalkulierter Lapsus, der punktuell eine überwachte Regression leistet, also kreativ den in Schach zu haltenden Wunsch offenbart. So wird etwa die Zote gerissen, das Warten auf die attraktive Versuchsleiterin sei weitaus schwerer gewesen als das Sitzen vor der Leckerei.

, Die (psychoanalytisch freilich kaum diskutierte) Coolness, welche die «Rührung durch die eigene Schöpfung» verweigert (vgl. Ulf Poschardt 2000, S. 117), also gewissermassen aus dem Marshmallow eine kleine Skulptur formt, unbewegt, aber auch nicht verlegen.

, Die Sublimierung, bei der das Triebziel ausgetauscht wird. Wie bei der Coolness erfolgt ähnlich zum Symptom anstelle des Verschlingens $z$. B. ein künstlerisches Verformen des Marshmallow, dieses Mal aber sogar mit 
Genugtuung, so als habe man eigentlich den ursprünglichen Trieb ausgelebt. Lacan definiert dieses Erhabenmachen als eine Art Reframing: eine Beförderung von Banalem zur «Würde» von etwas beherrschbar Triebhaften.

Der Erfinder des Marshmallow-Experiments, Mischel (2015), berichtet, dass viele Kinder sich spontan ablenkten, einfach die Augen schlossen, oder sich eben auch neue Aufgaben stellten. Wahrhaft sublimierend wäre der Rollentausch, selbst solche Versuche zu leiten, und dies mit der gleichen Lust wie früher beim Verzehr.

Fazit: Hemmung ist für Lacan nicht einfach der «normale» Bruder des verhaltenskreativen Symptoms. Sie kann quasi-psychotisch alles blockieren, aber auch zeitgeisttypisch «museumsreife» Shows abliefern, dabei in eine ohnmächtige Opferhaltung verfallen oder in Verlegenheit geraten. Denn die Angst vor der Selbstbegegnung, genauer vor der vom Subjekt selbst ins Objekt gesteckten Sinnstiftung (weniger Ödipus als individuelle Verlustaversionen) lauert potenziell in jeder Situation - diese ist daher niemals einfach «objektiv» gefährlich.

Der Ausweg der Hemmung, die Welt in musealer Starre aufzufassen, macht das Subjekt zum Hüter verlorener Träume und das Leben zum Besuch eines Friedhofs individuell «begrabener Begehren» ${ }^{10}$ - ein Ausdruck, den Lacan bei Ernest Jones findet.

\section{Lacan (1974-1975): Hemmung entsteht durch zu viel Imagination in der Sinnstiftung}

Zwölf Jahre und Seminare später tauchen Hemmung, Symptom und Angst bei Lacan wieder auf, jedoch scheinen das Neunerschema und auch der Akzent auf die Hinderung vom Tisch, er remixt schliesslich gerade seine eigene Lehre im Rahmen der mathematisch strengen Knotentheorie. Diese soll Antworten liefern, wie Reales, Imaginäres und Symbolisches zueinanderstehen. Sie fallen keineswegs zusammen mit Freuds Es, Ich, und Über-Ich. Abgekürzt als «RSI» lassen sie gar klanglich an "Häresie» denken, da sie nun - grob gesagt - auf die mentalen Register von Gefühlen, sprachlichen Gedanken und spiegelnden (liebenden und hassenden) Vorstellungen verweisen, wobei es eigentlich eher um das Unfühlbare, das Undenkbare und das Unvorstellbare geht.

Das RSI bezeichnet gewissermassen das Teamwork dreier Künstler zum Vergessenlassen der ontologischen Subjektschwäche - was soll ein Subjekt auch schon substantiell sein? An die Stelle des prekären Seins setzen die Drei unterschiedliche Repräsentationen der Objektursache: 
, Da gibt es den «Realisierer», der auf die harte Tour verstehen macht - und überdies stets verspätet. Man erkennt ihn in Einleitungen à la «Erst jetzt realisiere ich ...» Er lauert darauf, vermeintlich traumatisch erlebte Verluste zu reinszenieren (vgl. Anmerkung 10), und zwar immer dann, wenn die Erwartungshaltung an den Anderen und das ureigenste Verursachungsskript zusammenpassen.

, Daneben agiert der «Symbolisierer», der seinen Diskurs um verlustig gegangene treffende Worte herum organisiert.

, Last but not least will der «Imaginierer» immer da sein, wo er eigentlich gar nicht ist: Seine (Selbst-)Bildnisse vervollständigen disparate Elemente zu ganzen Gestalten (aber weniger originell als das Symptom).

Alle drei Experten bestimmter Nichtexistenz sind freilich selbst nichts, so lange sie sich nicht an die beiden jeweils anderen anlehnen können. Lacan gibt daher keinem dieser Künstler einen Vorrang, genauso wenig, wie ein Ring einer sogenannten «borromäischen» Verflechtung (vgl. Abbildung 2) dominant wäre. Wichtig ist zu verstehen, wie die Ringe auf diese borromäische, «Alle-oder-keiner»Art verflochten werden: Zunächst liegen nämlich zweie lose übereinander, bis ein dritter durch sie und ihre Schnittmenge geführt wird. «Lochmachen» nennt Lacan dies und sinniert darüber, welchen Unterschied es macht, wenn etwa der imaginäre Ring den Halt aller stiftet (also zuerst in den symbolischen eindringt oder sich stärker in ihn hineinschiebt - siehe den hellgrau markierten Keil).

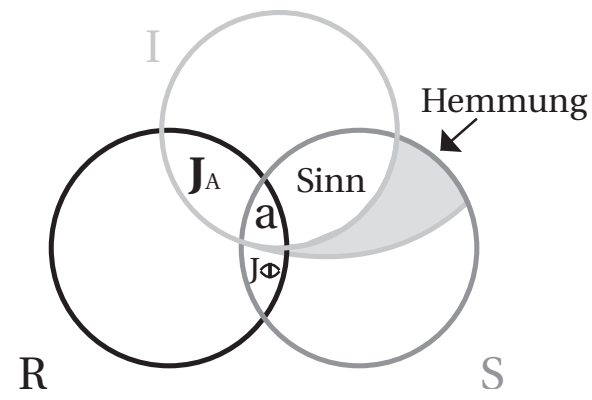

Die Schnittmenge zwischen Imaginärem und Symbolischem ${ }^{11}$ bekommt damit Schlagseite, so dass aus flüchtiger Bedeutung eine «arretierte» Sinnstiftung wird (sicherlich darf man dabei auch an Freuds idealerweise «freie», ungehemmte 
Assoziationen denken, oder aber an das Begehren, wie es metonymisch Ziele und Objekte wechselt). Damit liefert Lacan (1998) am 10.12.1974 ein Update der Hemmung: Sie erscheint nun als fixer Sinn oder gar fixe Idee, wo gewissermassen der Selbstbild-Künstler den Autobiographie-Künstler einengt und nun jede Abweichung gleich zum Verriss wird und schnell zu Schuldgefühlen führt. Eine weitere Form dieser Sinn-Hemmung können Wortspiele in Form eines Zurschaustellens des eingesetzten Klangmaterials sein.

In der finalen Sitzung des Seminars (14.05.1975) präzisiert Lacan, dass als Paradigma der Hemmung auch gelten könne, streng mathematischen Problemen mit imaginär geprägter, also ungenauer und v. a. moralisierender Normalsprache beikommen zu wollen. So mag es zwar als blumigere Umschreibung zutreffen, dass der Freudsche Neurotiker seine sexuelle Phantasie hemmt und stattdessen für die Galerie spricht. Lacan aber unterstreicht, dass der Gehemmte nüchtern betrachtet nur auf unbewusste Einschreibungen stösst und dann wie ein Mathematiklehrer beim Paraphrasieren von Tafelbildern in umständliche Ausflüchte hinein gerät (siehe die nicht alphabetisierbare, nicht transkribierbare «Knotenschrift» aus Abbildung 2). ${ }^{12}$ Keine von woher auch immer informierte Zensur muss als Hemmungsinstanz herangezogen werden - nein, was blockiert, ist die nicht 1:1 gelingende Übersetzung in eine fremde Darstellungskategorie, gewissermassen der den Autobiographen bedrängende Selbstbildnis-Künstler: Ein Sinnfilter, noch bevor dem Ich zugemutet würde, zu wissen, was ins Museum kommt.

Sieben Monate später, im Seminar Das Sinthome, bestimmt Lacan (2017) das «Unheimliche» ${ }^{13}$ als Teilmenge der Hemmung. Damit schliesst sich ein Kreis, denn schon 1962 ging es um die unheimliche Wiederkehr des Verdrängten. Und auch beim späten Lacan ist der Sinn da gehemmt, wo gerade das Heimische, das Eigenste als unheimlich erscheint (16.12.1975). Für Freud war Angst Zeichen der Verdrängung, analog dazu wäre für Lacan die Hemmung Zeichen der Wiederkehr des Verdrängten (diese Zeichendeutung hatte Freud nur für das Symptom in Erwägung gezogen).

Fazit: Hemmung ist nun weniger eine gefährliche Regungen aufs Eis legende Vorstufe derVerdrängung und Symptombildung, sondern ein Sinnphänomen. Auch ist es nicht das Ich, welches allzu luzide Erotisierbarkeitssignale empfängt, um dann eigene Funktionen zu hemmen, vielmehr suchen hier unterschiedliche psychische Stile der Verlustrepräsentanz eine Balance. Die Hemmung ist dabei das Zeichen eines Ungleichgewichts im Zusammenspiel des Benennens und des Bebilderns.

Dieses Mal müsste man stärker in den Kopf des Marshmallow-Probanden schauen, um zu beobachten, wie der Sinn stets auf falsche Weise produziert wird 
(vielleicht sich klein fühlen, über Wortspiele mit Marshmallows nachdenken müssen usw.).

\section{Die Psychoanalyse bleibt relevant - v. a. die Kulturphilosophie erklärt} das neoliberale Zeitalter mit Lacans hemmendem Genuss-Gebot

Ausgehend von der Slowenischen Schule um Žižek erklären heute auch Cederström/Spicer, Oswald-Rinner, Verhaege, Pfaller, Soiland sowie weitere Philosophen und Soziologen den aktuellen Zeitgeist mehr oder weniger direkt unter Bezugnahme auf die postödipale Psychoanalyse. Wo diese Quelle genannt wird, findet sich meist der Verweis auf Lacans Vermutung, dass das Über-Ich dieser Tage allgemein kein keusches «Geniesse nicht!», sondern ein mutiges, Enthemmung verheissendes «Geniesse!» befiehlt (exemplarisch dazu etwa Žižek, 1999). Mehr und mehr entziehe die westliche Kultur der Autorität bzw. dem «Herrn» die Macht, nach welchem noch der klassische Gehemmte oder Zwangsneurotiker sein Schema vom Anderen formte (inklusive der unspiegelbaren Objektursache).

Beschrieb Freud das hemmende Sich-Zwang-Antun noch als einen «Vorgang im oder am Ich» (Freud, 1926, S. 12), so verortet Lacan nun diese sonderbar befohlene, erzwungene Enthemmung in dem, was ihm einst das Stimmobjekt war: ${ }^{14}$ «Nichts zwingt jemanden zu geniessen, ausser dem Über-Ich» (Lacan, 2015, S. 9)

Im Seminar Encore von 1972-1973 erweist sich die kulturelle Befreiung als tückisch, geht eben doch gerade sie stark mit Zwang einher: Ohne dass die Hemmung hier strikt dem Begriff nach gebraucht würde, impliziert nun das Geniessen eine hemmende Rücksichtnahme auf den inneren Befehlsgeber. Zwar haben Gott und gottgleich waltender Herr historisch abgedankt, jedoch dominiert an deren Stelle die bürokratische Verwaltung, das Zwangsgeniessen der Wissensverwertung - leider ohne spürbare Enthemmung.

Warum auch sollte das Subjekt plötzlich neue Antworten haben, nur durch mehr Frechheit, durch das Aufbrechen u. a. religiöser Tabus oder durch die Zurschaustellung des Sexuellen (scheinbar gegenläufig zur Zensur schlüpfriger Gedanken)? Wo dieses Subjekt doch weiterhin, wie auch zuvor der Sprache unterliegt, für Lacan also grundlegend von der Frage getrieben ist, ob sein Objektursachen-Skript zum Willen des Anderen passt ...

«Nichts ist mehr erlaubt» lautet Lacans nüchterne Bilanz nach dem partiellen Ernstmachen der 68er-Generation mit dem «Tod Gottes»: die demonstrative Enthemmung gegenüber Trieblichem geht also einher mit noch mehr Hemmung. ${ }^{15}$

Genauso wenig wie die triumphale Vorstellung, dass es sich bei religiöser Unabhängigkeit ganz frei lebe, stimmt die Idee des Knechts, des Arbeiters, des 
Gehemmten, dass ein Machthaber oder ein Don Juan mehr geniesse (und man selbst nach dessen Beseitigung an seine Stelle käme).

Die Freudsche Logik des Über-Ichs («Je mehr ich gehorche, desto schuldiger bin ich») erscheint weniger paradox, versteht man den Abnehmer der work hard-, aber nun auch der play hard-Leistungen nicht als sättigbaren wohlwollenden Anderen, sondern als tyrannisches Über-Ich. Das Subjekt muss nun den Spass wie eine deontologische Pflicht exerzieren, unter einem malevolenten Blick, der überall das Ungenügen sieht.

Hat die Hemmung zuvor bereits im Ansatz das Ausüben von Trieben blockiert (und allenfalls Hinterhof-Vergnügen zugelassen), so überwindet nun jedermann den Türsteher, kommt dabei aber vom Regen in die Traufe: Denn im Zentrum der Marshmallow-Fabrik wird der Genuss optimiert, die neue Freiheit erlaubt auch beim Spasshaben nur effizientere Zielerreichung. Die Öffnung zum vorigen Jenseits des Herrn und der Genüsse macht die Irdischen, Machtlosen und Gehemmten mitnichten frei, sondern schafft auch noch das Geniessen aus guten alten Zeiten ab. Im vermeintlichen neuen Paradies gibt es ein Wiedersehen mit ihrem angestammten Joch: die Leistungslogik der Arbeit, für Lacan der «Mehrwert» des neuen (eigentlich aber je schon die Vernunft beschattenden) Geniessens. ${ }^{16}$

Fazit: Lacans Kulturanalyse einerWende zur Über-Ich-Akzentuierung wird von der Philosophie zustimmend aufgegriffen. Der verinnerlichte Befehl zu geniessen sorgt nicht für die erwünschte positive Enthemmung und Triebbefreiung, vielmehr drängen die Kulturstandards des bürokratischen, spätkapitalistischen Zeitalters dazu, das Begehren ausbrennen zu lassen und auf eine Zwangslust ganz neuer Qualität zu trimmen (diese erinnert letztlich aber doch auch an die Freudsche «Ersatzbefriedigung» im Symptom).

Im Marshmallow-Setting würden die Teilnehmer dieses Mal nicht autoritätshörig auf das Jenseits des zweiten Stück Mäusespeck spekulieren, sondern umstandslos das vor ihnen liegende Stück aufessen, allerdings mehr mit berechnender Geste als mit wahrhaft ungehemmtem Genuss.

Es bleibt zu resümieren, dass nach psychoanalytischer Auffassung die Hemmung eine Art sich Zwang antuender Verzicht aufs Trieb- oder wenigstens aufs Symptomausleben ist. Bei Lacan nicht so sehr aus Angst vor dem schamvollen Ertapptwerden oder vor einer Verdrängung, sondern vor zuviel Wissen um das Skript zur Mitverursachung von Objekten und Situationen - eine Rückkehr zu Freud durchaus, aber selektiv. 
Zuerst hatte Freud aus der Trieb-Not die Ich-Tugend der Hemmung gemacht. Dann deutete Lacan diese als ins Skript selbst eingehende Zurückhaltung vor offenerem Ausleben der Skriptanweisungen. Zuletzt findet sich in der gegenwärtigen Selbstoptimierungskultur ein Selbstzwang, so zu tun, als lebe man das Triebliche aus. So bekommt das Soziale mehr Gewicht, und erst so kann die Zwangsverwertung des Geniessens eine Sackgasse der neoliberalen Kultur anzeigen.

Zur Einstiegsfrage, wie die Hemmung psychoanalytisch zu bewerten sei: Bestenfalls kann man ihr eine gewisse Priorisierung von Wissen über die Triebe zugestehen, alles Weitere ist nur leere Geste. Trotz einer gewissen «Ersatzbefriedigung» erfüllt der Triebaufschub einer lustvoll «gewählten» Zurückhaltung zumeist gerade nicht den Zweck des sozialen Erfolgs. Auf kultureller Ebene befördert diese Hemmung einen Diskurs, der sich selbst aufzehrt.

Da Freud und Lacan beim Thema der Hemmung vergleichsweise skizzenhaft geblieben sind, verwundert es nicht, dass auch zur Behandlungstechnik wenig zu finden ist: Lacans gehemmter Patient Pierre Rey plauderte aus, dass er auch dann noch zahlen sollte, als er längst pleite war - ein drastischer Rauswurf aus der Komfortzone, während das Setting stur beibehalten wird (Vanheule, 2001, S. 126).

Dies würde aber wohl kaum, ebenso wenig wie auflockernder Humor, einen Über-Ich-Gehemmten stoppen. Hier hilft vielleicht nur noch der zweite Tipp Lacans: Denken, ohne Bildhaftem auf den Leim zu gehen, etwa indem man sich mit der Knotentheorie beschäftigt!

\section{Literatur}

Blanchot, M. (1947). À la rencontre de Sade. Les temps modernes, 25, 3. 577-612. Breuer, J. \& Freud, S. (1895). Studien über Hysterie (1. Auflage). Leipzig und Wien: Deuticke.

Freud, S. (1905c). DerWitz und seine Beziehung zum Unbewussten. GWVI(S. 1-269). Frankfurt: Fischer.

Freud, S. (1919h). «Das Unheimliche». GW XII (S. 227-268). Frankfurt: Fischer.

Freud, S. (1926d). Hemmung, Symptom und Angst. GWXIV(S. 111-205). Frankfurt: Fischer.

Jones, E. (1913). Papers on Psycho-Analysis. London: Ballière, Tindall and Cox. Harari, R. (2001). Lacan's Seminar on “Anxiety”. An Introduction. New York: Other Press.

Lacan, J. (2009). Das Seminar 10. Die Angst. Wien: Turia + Kant.

Lacan, J. (2015). Das Seminar 20. Encore. Wien: Turia + Kant.

Lacan, J. (1998). Das Seminar 22. R. S. I. (interner Arbeitstext). Bregenz: Lacan-Archiv. 
Lacan, J. (2017). Das Seminar 23. Das Sinthom. Wien: Turia + Kant.

Mischel, W. (2015). Der Marshmallow-Test: Willensstärke, Belohnungsaufschub und die Entwicklung der Persönlichkeit. München: Siedler.

Poschardt, U. (2000): Cool. Hamburg: Rogner \& Bernhard.

Vanheule, S. (2001). Inhibition: “I am because I don't act”. The Letter (Dublin), 23, $109-126$.

Widholm, R. (2008). Was nicht täuscht - Über den Begriff der Angst als Affekt (Magisterarbeit Universität Wien). https://core.ac.uk/download/pdf/11583214. pdf [10.02.2019].

Žižek, S. (1999). Enjoy!. Die Zeit, 34/1999. https://www.zeit.de/1999/34/Enjoy_/ komplettansicht [10.02.2019].

Abbildung 1: Zusammenschau Lacan 2009 (Sitzungen 25.06.1963, 03.07.1963) \& Widholm, 2008, S. 32

Abbildung 2: lacan-entziffern.de/borromaeischer-dreierknoten-mit-hemmung-3 (Rolf Nemitz)

\section{Anmerkungen}

1 Eine Autorität erklärt den direkt vor der lockenden Süssigkeit platzierten Kleinkindern, dass sie, sollten sie eine Viertelstunde der Versuchung widerstehen, gebilligt die doppelte Menge verschlingen dürfen. Vom Versuchsrahmen und vom kindlichen Alter der Probanden kann hier abgesehen werden.

2 Lacan verhandelt grösser dimensioniert und ohne Belohnung für den Verzicht das Beispiel eines unter Todesstrafe verbotenen Geschlechtsverkehrs - er hält wie Freud diese Art von verhängnisvollem Triebausleben für ein prinzipiell halbwegs mögliches Geniessen, Kant dagegen für eine völlige Unmöglichkeit bei moralischen Wesen.

3 Freud (1926d) meint die Hemmung durch verschiedene Anzeichen erkennen zu können: Lustlosigkeit, Schlamperei, häufige Unpässlichkeit, Abschweifung, Verlangsamung, Prokrastination, Unterbrechung sowie spätere Reuebekundung (S. $7 \mathrm{f}$.). Lacan wird später das Museale, die Zurückhaltung von individuellem Lebensausdruck hervorheben.

4 Fast könnte man fragen, ob die Abgrenzung des Symptoms gegen die Hemmung nicht ein wenig der eines vollwertigen Kunstwerks gegen den Kitsch entspricht, zumal sich auch die urhebergesetzliche «Schöpfungshöhe» am Grad der gehemmt so heikel erscheinenden Individualität orientiert. Die Innenwelt entscheidet hier: Impotenz etwa kann Anzeichen sowohl einer Hemmung als auch einer alternativen Symptomkreation sein.

$5 \quad$ Der Fall eines sich zwanghaft unter Plagiatsverdacht stellenden Denkgehemmten wurde für Lacan Anlass, beim behandelnden Ich-Psychologen Ernst Kris nur Eitelkeit zu finden, wo dieser didaktisch die ungerechtfertigte Selbstbezichtigung wie in einer Karikatur aufzeigen und wegargumentieren wollte - damit treffe er jedoch nicht die sexuell überdeterminierte unbewusste Überzeugung.

6 Die erwartbaren «Sollbruchstellen» bestehen für Lacan in Enttäuschungen durch das, was das Subjekt entwicklungspsychologisch gemäss seiner Erwartung von Anderen nie erwartet hätte:Verlassenwerden, strenge (Anstands-)Forderungen, Rollenzwänge und falsch Gesehen- oder Gehört-Werden. Dabei interpretiert Lacan die mit solchen Traumata assoziierbaren Körperöffnungen (Indizien für Abgeschnittenes) als Zonen, an denen sich besonders 
gut ein Körpergefühl mit Sinn aufladen lässt. Ein Sehnen nach Wiederverschluss resultiere je nach Besetzung in oraler Bedürfnisäusserung, in zwanghaftem Anspruch, in phallischem Geniessen, in Blick-Macht oder im Begehren einer Gewissensstimme. Der Eiertanz um die Frage nach dem Willen des Anderen, im Extrem die Furcht vor neuen Schnitten, führe oft zu einer Verlustangst, die sich die Nähe einfach «geniessend» erträumt - eigentlich aber müsse das Subjekt davor Angst haben.

$7 \quad$ Wobei unklar bleibt, wie sich diese acht Angst-Copings abheben von Lacans psychoanalytischem Basis-Diagnoseschema der psychischen Strukturen. Diese definiert er als Resultat der drei Freudschen Hauptabwehrtypen: die verdrängenden Neurotiker, die verleugnenden Perversen und die verwerfenden Psychotiker.

8 Hier ergäbe sich eine Verbindung der neurotischen Hemmung zum Vorgang der psychotischen Verwerfung, die wahrnehmbare Triebzumutungen am Objekt schlicht übersieht, sich nicht berechtigt fühlt.

9 Bei Lacan ist Acting-out weniger als bei Freud theatralische Abwehr gegen das Erinnern, als vielmehr ein Appell (v. a. an einen ungenügend intervenierenden Analytiker) oder gar ein gespielter Wahn.

10 Freud sprach noch von der Lustlosigkeit der Hemmung, Lacan kennt dies auch als eine ganz eigene Befriedigung: Gerade die sehr verbreitete psychische Struktur der Neurose lasse in der Variante des gehemmten Zwangsneurotikers nur leben im Warten auf den Tod des Anderen (ein individuelles Schema eines Herrn und Meisters), der in alle Objekte einen Willen nach Gehorsam einpflanzt. Andererseits liegt für den Zwanghaften Befriedigung auch darin, dass er selbst warten lässt, und sich so vermeintlich über die fortgesetzte Zurückhaltung (des Herzuzeigenden) die Zuwendung erhält.

11 «Begehren, das keinen direkten Ausdruck finden kann, wird nach innen gewendet. Aufkommende Furcht ist also eigentlich die Furcht des Patienten vor dem Ausbruch seines eigenen begrabenen Begehrens» (Jones, 1913, S. 191, eigene Übersetzung). Die verbotenen (trieblichen) Wünsche sind also nie ganz zu tilgen. Wo sie gehemmt wurden, taucht auch eine besondere Furcht auf - nicht einfach eine Begleiterscheinung der Verdrängung wie in der ersten Angsttheorie Freuds, sondern ein Zeichen der «unheimlichen» Wiederkehr des Verdrängten (vgl. Endnote 13).

12 Die Schnittmenge zwischen Symbolischem und Realem bestehe im stets neue unverbindliche Ziele ausfindig machenden phallischen Geniessen (treibt sich ein Keil des Symbolischen ins Reale entstehe das Symptom, ein besonderer Glaube an eine metaphorische Wunscherfüllung). In der Schnittmenge zwischen Realem und Imaginärem wiederum ergebe sich ein alternatives Geniessen, das eher weiblich-mystisch codiert ist und von einenden Verbindlichkeiten träumt (treibt sich ein Keil des Realen ins Imaginäre kommt Angst auf).

13 Im Zentrum der Schnittmengen findet sich nun das «a» (die hier bereits beschriebene Objektursache, also das Skript zur Verursachung von Bedeutung im Objekt). Dieses wird nun zum nur topologisch existenten Ort des in drei Geniessensformen aufgefächerten Sinnstiftens.

14 Natürlich würzt hier Lacan selbst seine topologische Darstellung, ein schon nicht mehr einfach Laute abbildendes Geschriebenes, mit imaginationsanregenden Worten: Was ihmgemäss den Neurotiker eher vom Verstehen seines Skripts der Objektursache abbringt, lässt nun auch sein Seminar eher gen geschwätziges Mathematikkolloquium abdriften. So regt er unter Hinzusetzung neuer Bilder an, einen Ring homolog zu einer unendlichen Geraden zu verstehen, wie sie wiederum das «echte» Gegenstück zum «falschen Loch» eines Schuhbinde-Schleifenknotens sei.

15 Freud (1919h) spezifiziert mit dem Unheimlichen eine besondere Angst, nämlich das Gefühl, das bei der Wiederkehr des Verdrängten aufkommt (S. 317).

16 Früher nannte Lacan das Über-Ich im Zusammenhang mit der «Objektursache des Begehrens im Anderen» (12.06.1963), eine Abspaltung oder Verwerfung aus dem Symbolischen, jetzt ist es allgemein das Zwänge auslösende "Geniessen des Anderen» 
(21.11.1972). Dass die Hemmung auf Kulturebene nicht unbedingt an ein tyrannisches und teils auch zu Gewalttaten führendes Über-Ich gebunden sein muss, könnte evtl. an Japan belegt werden, wo die Hemmung in Umfragen stets Spitzenwerte erzielt, aber weit weniger Gewaltdelikte als im Westen gezählt werden - ohnehin sei in Japan mit einem ganz anderen Unbewussten zu rechnen, meint Lacan.

17 Das vorig Private, demgegenüber Gott oder Über-Ich auch mal ein Auge zudrückten, wird nun vom Geniessen eines nie zu sättigenden Anderen erfasst. So wird auch das Ausleben des Geniessens zum «stehenden Ausdruck». Das Geniessen ist nicht mehr, was es war, nimmt Warencharakter an, zieht Kalkulationen auf sich.

18 Lacan schöpft mit dem Begriff «plus de jouir» aus, was ihm das französische «plus de» anbietet: es meint sowohl ein «Mehr» an Lust (ein Extra, etwas Neues) als auch ein «NichtMehr» (die darin abgeschaffte alte Lustökonomie ergaunerten Geniessens).

\section{Angaben zum Autor}

Andreas Spohn, geb. 1972, Psychoanalytiker PSZ, Arbeit in Zürcher Praxis und in Angehörigenberatung (Umfeld Sucht); Interessen:Verbindungen zwischen Philosophie und Psychoanalyse. 